\title{
Psychosocial Aspects and Quality of Life in Cancer Patients undergoing Surgery
}

M. Bakos (Marian Bakos)1 , T. Jankovic (Jankovic Tomas)¹, V. Krcmery

(Madimir Krcmery)2 ${ }^{2}$ M. Vidiscak (Marian Vidiscak) ${ }^{3}$, S. Durdik (Stefan Durdik) $)^{4}$

${ }^{1}$ Department of Surgery Faculty Hospital Nitra, SK.

Original Article

${ }^{2}$ St. Elizabeth University, Slovakia and Royal College of Physicians

Edinburgh, UK.

${ }^{3} 1^{\text {st }}$ Department of Surgery, Faculty of Medicine, Slovak Medical University in Bratislava, SK.

${ }^{4}$ Department of Oncological Surgery, Oncology, Faculty of Medicine, Slovak Medical University and Institute of St. Elizabeth, Bratislava, Sk.

\section{E-mail address:}

info@fnnitra.sk

\section{Reprint address:}

Marian Bakos

Faculty Hospital Nitra

Department of Surgery

Nitra

Slovakia

Source: Clinical Social Work and Health Intervention

Volume: 12

Issue: 4

Pages: $78-84$

Cited references: 24

\section{Reviewers:}

Zofia Szarota

Pedagogical University of Cracow, PL

Roberto Cauda

Institute of Infectious Diseases, Catholic University of the Sacred Heart, Rome, IT

\section{Keywords:}

Breast Cancer. Quality of Life. Psychosocial Morbidity. Psychosocial Aspects.

\section{Publisher:}

International Society of Applied Preventive Medicine i-gap

CSWHI 2021; 12(4): 78 - 84; DOI: 10.22359/cswhi_12_4_10 @C Clinical Social Work and Health Intervention

\section{Abstract:}

Introduction: Quality of life in cancer patients and probably also long term survival is negatively affected with fear of the recurrence of cancer and consequences of aggressive therapy. This is the reason for anxiety, depressions, and frustration which are accompanied by cognitive, emotional and behavioral disorders in their physical, relationship, sexual and social functioning. The aim of our study is to analyze the dynamics of evolution of the psychosocial loads and qualitative changes its symptomatology in cancer patients who survival 1-4 years after surgery. 
Material and methods: For assessment of psychosocial morbidity's in measure in surgery patients standardized questionnaires of European Organization for Research and Treatment of Cancer- questionnaire EORTC QLQ-C30.3 (Quality of LifeC.30.3) and its module EORTC QLQ- BR23 were used.

Results: The degree of psychosocial mortality in survival patients one year after MRM in our group clearly demonstrated that these patients suffered because of consequences of psychosocial loads. It results from fear of recurrence of cancer and next functional, emotional, cognitive and social disorders without regard for surgery range. The degree of psychosocial loads in patients after MRM is still more than 4 years after end of treatment. Results show, that after the end of treatment there are many changes in their lives - anxiety and depressions can lead to psychical lability because of higher doubts, nervousness, irritability, helplessness and loneliness in their sufferers. Conclusion: Out-patient psychosocial interventions and preventions because of negative impact of ongoing and changing psychosocial loads becomes necessary. It means that there is negative impact on quality of life; of long term cancer remission and survival of patients after surgery.

\section{Introduction}

Breast carcinoma $(\mathrm{BC})$ is the most frequent oncological disease in Slovak women and its incidence is still rising. Mortality for breast malignity decreased about $8 \%$ in Slovakia between 2011 and 2016. Although in this period, the incidence of these diseases increased about $23 \%$. Thanks to better screening it identifies more diseases in early stages (more than $75 \%$ of breast cancer is diagnosed in stages I and II) and the time of survival is longer. It is clear that the number of women with breast cancer with long term survival is increased (1).

Despite the increased trend of malignant disease's incidence in many European countries, mortality has decreased and has increased the number of patients who survival more than 5 years. Breast carcinoma is the most frequent carcinoma in women and the second most frequent disease (after colon cancer) in the population. According of WHO, in the last two decades in Europe there is a decrease in mortality because of breast carcinoma. Significant progress in this aspect is thanks to better screening of early stages of carcinoma. We use ultrasonography and mammography in routine clinical practice; better methodology; better strategy of treatment (2).

In Europe, recent meta-analyzes of breast carcinomas shows a 5 year period of survival in treated patients reached $65-82 \%$ depending on the range and aggressiveness of a cancer. From all types of cancers 5 year survival in breast cancer patients is $23 \%$ world-wide $(3,4)$. This means there are 9.5 million women who must counter diagnosis of breast cancer and have to learn to live with this disease. They have to live with this progressive disease also in a no relapse period after undergoing difficult and mutilating curative treatment (5). In Slovakia, the number of survival patients increase about 1,200 every year by more than 2,000 new diagnostic breast carcinomas (2,198 cases in 2016-17. 7\%). So finally, in a 5 year period there are 6,000 survival patients who have to fight with the consequences from diagnosis and treatment (1).

In a no relapse period, patients have to deal with consequences of aggressive, radical surgery (pain, asymmetry of breasts, shoulder fibrosis, edema of upper extremities) and subsequent adjuvant cyto-destructive therapy (fatigue, insomnia, weakness). In addition, psychosocial loads potentiate fear for recurrence of cancer disease; loss of femininity; feelings of powerlessness; loneliness; changes in sexual sphere; decrease of physician, family and social functionality. Despite this, there is a group of patients who are balanced with disease and positive fight against it. There is a group of patients with severe psy- 
chosocial loads which means that their behavioral risk profile decreases total quality of life and which is leading into social isolation; apathy; loss of life interest and its filling. Previous analyses of psychosocial morbidity are basically focused on two fundamental parameters: anxio-depressive condition resulting from diagnosis and treatment of cancer; and second are manifestations of functional decrease in the cognitive-behavioral sphere. The aim of our study is to analyze the degree and dynamic of evolution of psychosocial morbidity and its consequences on quality of life in cancer patients who underwent radical mastectomy (MRM) with subsequent adjuvant radiotherapy 1-4 years after surgery with standardized questionnaire research (1).

\section{Material and methods}

In the study were included and evaluated $\mathbf{1 0 5}$ patients (T2b-T3) with breast carcinoma who survival in a 1-4 year no-relapse period after MRM (average year $60.5 \pm 14.0$ ). A distribution according of marital status was: single $(6 \%)$; married or in partnership (61\%); divorced (24\%); widowed (9\%). 95\% women mentioned religion affiliation. Distributions in working condition were: employed (27\%); self- contained (14\%); unemployed (2\%); housewife $(33 \%)$; unformed job $(2 \%)$; retiree $(22 \%)$. Treatment of the disease was: surgery $(96 \%)$; radiotherapy $(35 \%)$; chemotherapy (77\%); hormonal treatment (41\%). Range evaluation of psychosocial loads and quality of life of 1-4 years survival patients (1year \pm 3.7 months) and 4 years ( \pm 4.2 months $)$ after the end of treatment was analyzed using by standardized certificated questionnaires.

\section{Multidimensional questionnaires}

In this study we used standardized questionnaires of the European Organization for Research and Treatment of Cancer - questionnaire (EORTC). They were an official Slovak version. 115 patients answered questions in the questionnaire during regular control examination in the Breast Surgery Outpatient Department.

\section{EORTC OLO- C30 with module OLO- BR23 - European Organization for Research and Treatment of Cancer Quality of Life Questionnaire-Breast cancer Module}

Questionnaire EORTC QLQ- C30 with module for women with breast carcinoma are questionnaires used for clinical research $(2,3)$.

EORTC QLQ-C30.3 questionnaire (6) includes questions about 5 functional scales which evaluate physical; family, emotional; cognitive; and social functions; 3 functional scale about clinical symptomatology and separate 7 items scale evaluate total quality of life of oncology patients.

QLQ- BR23 contains 23 items and is for patients after mastectomy and for every method of treatment. Questionnaire EORTC QLQ-B23 is module of previous questionnaire and it is for evaluate of treatment's side effects and next emotional dysfunctions. Both questionnaires are based on a scoring system of actual feelings of psychosocial deterioration. It used 4 items Lekert's scale (absolutely no; a little; enough; very). Calculation of gross score of linear transformation into the scale of 1-100 and statistical evaluation were done according EORTC QLQ-C30.3 manual. Validity of results was statistical verified.

A questionnaire creates functional scale: physical self-image; sexual function; satisfaction with sexual life; fear of future health; and four symptomatic scale side effect of treatment; symptoms connected with arm of operating side; symptoms connected with treated breast; symptoms connected with loss of hair. Items (for example: Did your arm hurt?) are evaluated on a 4 points scale from 1 (absolutely no) to 4 (very). Answers responded to status during the last 7 days and last 4 weeks in the case of evaluating sexual life. Functional scales evaluate: zone of physical function; do the routine work; emotional zone; cognitive; social zone. In emotional scales are evaluated: fatigue; dizziness; pain; individual items evaluate: dyspnea; insomnia, loss of taste; obstipation; diarrhea; financial problems because of treatment. Items (for example:, Were you tired? ) are evaluated on a 4 points scale from 1 (absolutely no) to 4 (very). Items, which are connected with HRQOL (for example: How do you rate your health during last week?) are evaluated on a 7 points scale from 1 (very bad) to 7 (excellent) (6). 


\section{Questionnaire SLDS- BC - Satisfaction with Life Domains Scale for BC}

SLDS- BC is a 32 items self-assessment questionnaire which evaluates HRQOL in different phases of disease, from determination of diagnosis, treatment, until recovery. Items are evaluated on a 7 points scale. Patients choose pictures of faces (emoticons) from happy (1) to incredibly sad (7). There are 5 zones evaluated:

1. social functions (employments, relationships, overall satisfaction)

2. appearance (look, weight, life energy)

3. physical function (physical ability, healthy complication of disease and pain)

4. communication with medical stuff (physicians, nurses, other medical stuff)

5 . spirituality zone (important and benefit of individual spirituality in treatment) (5).

Questionnaires applicable for physical selfimaging BIBCQ - Body Image after BC

BIBCQ is a 53 items questionnaire about long-term influence of breast carcinoma treatment on physical self-imaging of women. It includes 6 optional items for women with both breasts and 2 optional items for women after one or both breast mastectomy. Items create 6 scales:

1. vulnerability (sensitivity of disease, for example: I' $\mathrm{m}$ afraid about my body?)

2. physical stigma (need to hide own body, for example: I cannot look at my body, on my scars after surgery..,,)

3. restrictions (feelings of physical ability, for example: I feel tired during the day.)

4. rating of body (satisfaction with body and look, for example: I am fine with my body.)

5. visibility (doubts about physical changes, for example:,I feel, that they are looking on my chest...,.)

6. evaluation of upper extremities on the operated side (symptoms of hand and its look, for example: I have pain of my hand.)

Items are evaluated on a 5 points scale from 1 "absolutely no" to 5 "absolute agreement ", or from 1 "never / almost never "to 5"always/ almost always ". This questionnaire is useful and undemanding (5).

\section{MAS - Mastectomy Attitude Scale}

Questionnaire MAS has 33 items, and it is done within clinical study. It is oriented on women's position after mastectomy. It reviews 7 zones: negative feelings about mastectomy; sexual sphere; satisfaction with look; life style; hiding of operated breast; honesty to theme of mastectomy; sense of importance of mastectomy. Individual items are evaluated on a 4 points scale from 1 " don’t agree" to 4 "agree" (5).

\section{Results}

\section{Quality of life in survival patients with breast carcinoma}

For comparison of overall quality of life in patients with $\mathrm{BC}$, who survival 1 and 4 years after MRM a 7-point scale (very bad to very well) is used. This data show that quality of life in the fourth year after MRM is not changed in all against first years survivals. These patients evaluate their quality of life as bad till tolerable (score 1-5, average 3.7 vs 3.5). In all groups there is no patient after MRM who evaluate her quality of life as very well (score 7).

\section{Psychosocial determinates of life quality in survival patients with breast carcinoma}

Comparison of patient's psychosocial loads one year after MRM according to emotional parameters responded to low score of life quality. Parameters of sexual function; feelings of femininity loss; afraid of job; feelings of uncertainty; loneliness; afraid of future were very intensive after MRM. There are other items which are scored in patients after MRM: emotional dysfunction with anxiety; nervousness; irritability; financial doubts; clinical symptoms include fatigue and pain. In the period of four years survival after treatment we can observe divergence of psychosocial morbidity symptoms.

Our results show that the degree of psychosocial loads and quality of life is dynamic and qualitative differentiated process - in some parameters increased manifestations of emotional and social disorders. The outcome of this changed quality of life is: feeling of loss my ,, me, loss of personality value; change of personal features; changes in partnerships; emotional disorders. All these changes result in: evolution risk behavioral profile; social isolation; loss of ability to face cancer. Our analyses confirm that more than $70 \%$ of survival patients with breast carcinoma progressively emulate with consequences of cancer. 
The feeling of loss of femininity decreases; feelings of loss sexual function disappear; physical and family functions are emulated. On the other side, in many of these patients: increased feeling of doubt; nervousness; irritability and it is graduated. This process of dynamic psychosocial loads is potentiated by permanent weakness and clinical symptoms which are undesirable consequences of treatment (pain, arm fibrosis, gastrointestinal disorders, insomnia). Risk behavioral profile of survival patients which is evolving mostly in young patients and psychically unstable women contains disorders in cognitive and behavioral spheres.

\section{Psychosocial support}

Sources of social support are in Table 1. Most patients $(\geq 75 \%)$ wanted and got social support from physicians and friends/family. Other sources of social support were not so wanted and rarely gotten. All types of social support were for women extremely helpful. The most helpful sources were physicians, nurses, and friends/members of family. The right column of Table 1 is related only for women who got only this type of support. Between these variables of social support and summary score EORTC QLQ-C30 there were not statistically significant relation.

Table 1 Frequency of social support

\begin{tabular}{|l|c|c|c|}
\hline & $\begin{array}{l}\text { Support } \\
\text { required }\end{array}$ & $\begin{array}{l}\text { Support } \\
\text { accepted }\end{array}$ & $\begin{array}{l}\text { Support } \\
\text { successful }\end{array}$ \\
\hline Physician & $72 \%$ & $79 \%$ & $97 \%$ \\
\hline Psychologist & $34 \%$ & $15 \%$ & $92 \%$ \\
\hline Social worker & $8 \%$ & $3 \%$ & $66 \%$ \\
\hline Clergy & $18 \%$ & $9 \%$ & $86 \%$ \\
\hline Nurse & $29 \%$ & $7 \%$ & $86 \%$ \\
\hline $\begin{array}{l}\text { Friends / } \\
\text { Family }\end{array}$ & $70 \%$ & $78 \%$ & $98 \%$ \\
\hline
\end{tabular}

\section{Discussion}

Similarly, as in other European countries, patients with breast carcinoma are the largest group of survival persons with carcinoma diseases. 2,000 new diagnosed carcinomas every year means, that in 5 million population every year an increase number of patients who live in fear and doubt for the future. They have feelings about threat of untimely death; loss of femininity; frustration; other emotional disparities which funda- mentally decrease quality of life. Ganz et al. (3), Holzner et al. (7) and other authors in extensive clinical studies show, that psychosocial loads from diagnosis and treatment of breast carcinoma decrease until one year after the end of treatment, but in some part of patients still for long time. Psychosocial morbidity has negative influence on quality of life and negative influence for average survival period (8). Patients, who live in long time doubt, frustration and depression, very often are isolated from natural social bonds and stay in social isolation. $(9,10)$. All these facts have negative influence on their quality of life; ability to face cancer with its negative consequences (1). Healthcare providers give emphasis on clinical parameters of healthcare (success of treatment; side effect of curative treatment; average period of survival). They don't give attention to psychological and social consequences of cancer in its all over trajectory - from diagnosis until end of treatment and follow dispensaries. Anxiety and depression are normal, part of the cancer process for them

Patients are dependent for emotional support which they search for in family or psychologist. In contrast with us, in the most advanced European countries, also in USA or in Australia, there are complex psychosocial systems of healthcare. Specialized oncological social workers provide psychosocial support. They are targeted, professionally prepared and are part of a caring team (11). European studies from Italy (12), Greece (13), Spain (14) and Turkey (15) found worse levels of QoL in breast carcinoma patients. While a study from Holland had abetter level QoL. (16). A moderate score form a Brazil study (17) was like our moderate score.

One of the possible reasons, why there were reported only mild damages of life quality, can be the bias against selection. Women from high society were more included, because they had better chance of treatment in hospital, which was in study. It can be a reason for better quality of life. Another Columbian study of breast cancer patients found that a higher degree of education is connected with higher a quality of life (18). Treatment modes didn't have statistically significant influence on QoL. Extensive research, with more than 1,000 breast cancer patients (19), didn't demonstrate significant differences in quality of life because of the kind of treatment: 
radiotherapy, chemotherapy or hormonal therapy. This does not mean that these therapeutic modalities don't have an influence on quality of life.

There is optimism connected with QoL. Every function scale were positive correlated with subscale of optimism LOT-R and 6 from 9 scales and items of symptoms were negative correlated. The highest correlation was found by generally evaluated health/quality of life $(r=0,31)$. These positive associations between optimism and quality of life are demonstrated also in the common population (20). Religions and personal features were proved as mediator of relation between optimism and prosperity (21). We were not able to test the role of these mediators in our study. Except for correlations with QoL it is interesting that average score of patient optimism was not lower, but in actuality was higher than in the common population. Also, other studies demonstrated similar results. Average score of LOT-R in the groups of patients with breast carcinoma was between 16.2 and 16.9 (22, $23)$, which is higher than the average score in common European population. A German study found that patients with cancer want and really get social support from physicians and family/friends. Other specialists (psychologists, social workers, pastors) are engaged less (24). Physicians must know that they are not only specialists for patients, but patients hope for psychosocial support many patients were pleasured because of physicians' support. But only half of patients who want this support actually got it. It is necessary to reconsider and evaluate barriers to another possibility of care. Integration of short time forms of monitoring of life quality in daily clinical routine can be a highly effective form for detecting physical and psychical disorders and their monitoring in time.

\section{Conclusion}

The degree of psychosocial mortality in survival patients one year after MRM in our group clearly demonstrated that these patients are suffering because of consequences of psychosocial loads. This results from fear of recurrence of cancer and next functional, emotional, cognitive, and social disorders without regard for surgery range. The degree of psychosocial loads in patients after MRM is still more than 4 years after end of treatment. Results show that after end of treatment there are many changes in their life: anxiety and depressions can lead to psychical lability because of: higher doubts; nervousness; irritability; helplessness; loneliness in their sufferers. It is clear, that without adequate psychosocial intervention there is still symptomatology of psychosocial morbidity. It means that quality of life is inferior; the results of treatment are worse; ability to face to cancer is less. Results of our study confirm urgent need for prevention of psychosocial distress which relates to cancer with adequate forms of professional psychosocial interventions.

\section{References:}

1. VALOVICOVA E (2018) Incidence of cancer in the Slovak Republic. Oncology education for universities. 2. ed. Bratislava: League Against Cancer,. ISBN 978-80-89201-38-9. pp. 22-23s.

2. AUTIER P, AIT OUAKRIM D (2008) Determinants of the number of mammography units in 31 countries with significant mammography screening. Br J Cancer; 99(7): pp. 1185-1190.

3. GANZ PA, HAHN EE (2008) Implementing a survivorship care plan for patients with breast cancer. J Clin Oncol; 26(5): pp. 759767.

4. MONTAZERI A (2008) Health-related quality of life in breast cancer patients: a bibliographic review of the literature from 1974 to 2007. J Exp Clin Oncol; 27: 32. 21. N

5. LIPSCOMB J, DONALDSON MS, HIATT RA (2004) Cancer outcomes research and arenas of application. J Natl Cancer Inst Monogr; 33: pp. 1-7.

6. AUTIER P, AIT OUAKRIM D (2008) Determinants of the number of mammography units in 31 countries with significant mammography screening. Br J Cancer; 99(7): 1185-1190.

7. HOLZNER B, KEMMLER G, KOPP M et al. (2001) Quality of life in breast cancer patients - not enough attention for long-term survivors? Psychosomatics; 42(2): pp. 117123.

8. KROENKE CH, KUBZANSKY LD, SCHERNHAMMER ES et al. (200) Social networks, social support, and survival after breast cancer diagnosis. J Clin Oncol; 24(7): pp. 1105-1111. 
9. IASWR (2003).Report to the National Cancer Institute. Social Worker's contribution to research on cancer prevention, detection, diagnosis, treatment and survivorship. Nat Cancer Inst: p. 44.

10. MICHAEL YL, BERKMAN LF, COLDITZ GA et al. (2002) Social networks and healthrelated quality of life in breast cancer survivors: a prospective study. J Psychosom Res; 52(5): pp. 285-293.

11. HODGKINSON K, BUTOW P, HUNT GE et al. (2007) Breast cancer survivors' supportive care needs 2-10 years after diagnosis. Support Care Cancer; 15(5): pp. 515-523.

12. SPATUZZI R, VESPA A, LORENZI P et al. (2016) Evaluation of Social Support, Quality of Life, and Body Image in Women with Breast Cancer. Breast Care;11: pp. 28-32.

13. KONTODIMOPOULOS N, NTINOULIS K, NIAKAS D (2011) Validity of the Greek EORTC QLQ $\square$ C30 and QLQ $\square$ BR23 for measuring health related quality of life in breast cancer patients. European Journal of Cancer Care, pp. 354-361.

14. ARRARAS JI, MANTEROLAA, ASIN G et al. (2016) Quality of life in elderly patients with localized breast cancer treated with radiotherapy. A prospective study. Breast, 26, pp. 46-53.

15. DEMIRCI S, ESER E, OZSARAN Z et al. (2011) Validation of the Turkish versions of EORTC QLQ-C30 and BR23 modules in breast cancer patients. Asian Pacific Journal of Cancer Prevention, 12, pp. 1283-1287.

16. BANTEMA-JOPPE EJ, DE BOCK GH, WOLTMAN-VAN IERSEL M (2015) The impact of age on changes in quality of life among breast cancer survivors treated with breast-conserving surgery and radiotherapy. British Journal of Cancer, 112, pp. 636-643.

17. EVANGELISTAAL, MONTEIRO SANTOS EM, DO SOCORRO MACIEL M (2016) Associations of quality of life, physical activity and mood states in women with breast cancer treated with curative intent. Applied Research in Quality of Life, 11, pp. 445-459.

18. ZAPATA CS, ROMERO HG (2010) Quality of life and associated factors in women with breast cancer in Antioquia, Colombia. Revista Panamericana de Salud Publica, 28, pp. 9-18.
19. WALDMANN A, PRITZKULEIT R, RASPE H, KATALINIC A (2007) The OVIS study: health related quality of life measured by the EORTC QLQ-C30 and-BR23 in German female patients with breast cancer from Schleswig-Holstein. Quality of Life Research, 16, pp. 767-776.

20. SCHOU-BREDALA I, HEIR T, SKOGSTAD L et al. (2017) Population-based norms of the Life Orientation Test-Revised (LOT-R). International Journal of Clinical and Health Psychology, 17, pp. 216-224.

21. MATTHEWS EE, COOK PF. (2009) Relationships among optimism, well-being, selftranscendence, coping, and social support in women during treatment for breast cancer. Psycho-Oncology, 18, pp. 716-726.

22. GARNER MJ, MCGREGOR BA, MURPHY KM (2015) Optimism and depression: a new look at social support as a mediator among women at risk for breast cancer. Psycho-Oncology, 24, pp. 1708-1713.

23. THIEME M, EINENKEL J, ZENGER M, HINZ A (2017) Optimism, pessimism and self-efficacy in female cancer patients. Japanese Journal of Clinical Oncology, 47, pp. 849-855.

24. ZENGER M, FINCK C, ZANON C et al. (2013) Evaluation of the Latin American version of the Life Orientation Test-Revised. International Journal of Clinical and Health Psychology, 13, pp. 243-252. 